\title{
Market Informational Inefficiency, Risk Aversion and Quantity Grid*
}

\author{
Jean-Paul DECAMPS ${ }^{\dagger}$ and Stefano LOVO $¥$
}

April 7, 2003

\begin{abstract}
In this paper we show that long run market informational inefficiency is perfectly compatible with standard rational sequential trade models. We consider a financial market where: (i) tradable quantities belong to a quantity grid; (ii) traders and market makers do not have the same degree of risk aversion. We show that as soon as traders' beliefs do not differ too sharply, the equilibrium of the economy is unique and non-informative. Thus, the market cannot completely aggregate private information and long term mispricing occurs almost surely. We explain the ambiguous role of the quantity grid in exacerbating or mitigating market inefficiency. We show that stock splits can improve the information content of the order flow and consequently increase price volatility. (JEL G1, G14, D82, D83)
\end{abstract}

Keywords: Informational efficiency, quantity grid, stock splits.

${ }^{*}$ We would like to thank Bruno Biais, Thierry Foucault, Christian Gollier, Ulirch Hege, Jacques Olivier and Jean-Charles Rochet for insightful conversations and valuable advice. We would also like to thank the seminar participants at Turin University and the participants to the Sixth Toulouse Workshop in Finance for useful comments and suggestions. Of course all errors and omissions are ours.

${ }^{\dagger}$ GREMAQ-IDEI Université de Toulouse 1, 21 Allee de Brienne, 31000 Toulouse, France, corresponding author, e-mail: decamps@cict.fr

${ }^{\ddagger}$ HEC, Finance and Economics Department, 1 Rue de la Liberation, 78351, Jouy en Josas, France, e-mail: lovo@hec.fr. 


\section{Introduction}

One of the central roles of financial markets is to provide information about asset's fundamentals through the price system. After the recent collapse of companies that were commonly considered, and priced, as worthy and safe, investors questioned the capacity of the market to perform this crucial task. For example, it is now clear that Enron's fall was long time coming and that pieces of information about the company's problems were spread among agents. Then, why have the price of Enron's shares remained for so long far above the company's fundamental value?

In financial economics, it is generally accepted that when it is possible to observe the actions of a sufficiently large number of rational investors, trading prices eventually incorporate all the information available in the economy. ${ }^{1}$ This happens because each investor's action discloses, at least partially, the investor's private information on fundamentals. This information is incorporated into the trading prices. Thus, by observing these prices, it is ultimately possible to infer all the relevant private information that is dispersed among market participants. In other words, in the long run, the market is informational efficient. For this reason, some practitioners and financial economists attributed mispricing episodes to market exuberance or investors irrationality.

In this paper we show that the price system can fail to aggregate information even in the presence of an infinity of privately informed rational investor. Thus, long term mispricing is perfectly compatible with agent's rationality. To this purpose, we generalize a standard financial market model to consider two features of actual financial markets: tradable quantities belong to a quantity grid (in particular it is impossible to trade fractions of a share); traders and market makers do not have the same degree of risk aversion. We show that when these two factors are taken into account, then market is not informational efficient in the long run. In other words, surprisingly, in the long term the private information regarding the asset fundamental value cannot be completely

\footnotetext{
${ }^{1}$ This is a textbook result in financial economics. See for example O'Hara (1995) or Biais, Glosten and Spatt (2001) for a recent review of the financial microstructure literature.
} 
incorporated into trading prices. As in the model we consider, trading prices are equal to the expected value of the risky asset given the history of trades, we can measure the long-term-mispricing with the distance between the trading prices in the long run and the expected value of the asset for someone who has the combined knowledge of all traders in the economy. We show that in general this distance cannot vanishes, and that the resulting "long term pricing error" can be large.

More precisely, the model we consider is a sequential trade model similar to Glosten and Milgrom (1985) and Glosten (1989): in each period risk neutral market makers quote a price schedule for a risky asset. Given the price schedule, informed risk averse traders choose the size of their trade. The difference with the existing literature is that we bring together on the one hand the existence of a grid for tradable quantities, on the other hand, discrepancy in risk aversion between dealers and traders.

In order to have an intuition of our result, notice that a risk averse privately informed trader's order includes two components: an informational component and an inventory component. The first component comes from the trader's informational advantage given by his private information on the asset's fundamental. The latter component follows from the trader's risk aversion and is not related to the asset's fundamental. Note that when the past history of trades provides a sufficiently precise information about the asset's fundamental, then an additional partially informative private signal will affect slightly a trader's belief. Thus, as a trader can demand only discrete quantities of the asset, a small change in his belief will in general not be sufficient to affect his demand and so, eventually all traders' demands will only reflect their inventory components. From this point on, the flow of trades will no longer be informative, the social learning process stops and trading prices will be bounded away from market fundamental.

In short, when the market is quite sure about the asset's fundamental value, the equilibrium of the economy is unique and such that the flow of trades does not provide any new information because orders only reflects traders' inventory concerns. Moreover, if the learning process stops when the market is quite sure about the asset's fundamental but in a completely wrong direction, then prices will be trapped far away from the asset 
fundamental value, and consequently the long term pricing error will be large.

Other papers in the financial microstructure literature have considered separately the discrepancy in risk aversion and discrete trading without obtaining informational inefficiency. For example, in Glosten and Milgrom (1985) or Easley and O’Hara (1992) traders can only trade discrete quantities (buy 1 asset, sell 1 asset, no trade) but in these models both market makers and informed traders are risk neutral, so trades are always informative. In Glosten (1989), Vives (1995) and Biais et al. (2000) risk neutral market makers face risk averse informed traders, but these models assume that it is possible to trade a continuum of quantities of the asset so that even a tiny information component can affect the trader's order, and for this reason the order flow is always informative. Thus, our contribution is to show that the combination of differences in risk aversion and discrete trading stops the learning process at wrong prices and this generates informational inefficiency. Moreover, we show that long-run-mispricing will increase with traders' risk aversion and with the fundamental's volatility that cannot be explained through private information. It will decrease with the precision of traders' private signals. This recalls the findings in Vives (1995) where the speed of convergence of prices to fundamentals is negatively affected by traders' degree of risk aversion and positively affected by the precision of private information of agents. Differently from Vives, we show that prices cannot converge to fundamentals as the speed of convergence is zero as soon as traders' beliefs do not differ too sharply.

Our main result is in line with the theoretical literature on "herd behavior" that proves that sequential interaction of rational investors can generate rational imitative behavior and this prevents agents from learning the market fundamental. ${ }^{2}$ However, most of the results in this literature are based on the assumption that transaction prices are exogenously fixed and are not affected by the information provided by past trades. Therefore, the herding literature cannot be directly applied to stock markets, and it is clearly unfit to study the issue of the informational content of prices. Herding in financial markets has been studied by Avery and Zemsky (1998). They introduce multidimensional

\footnotetext{
${ }^{2}$ See Chamley (2001) for an extensive study on the causes of rational herding.
} 
uncertainty in a Glosten and Milgrom (1985) style model and show that herding and short-run-mispricing can occur. However, as well as in Glosten and Milgrom (1985), in the long term trading prices converge to the fundamental value of the asset.

Long term mispricing in the presence of endogenous price is obtained by Lee (1998). He shows that information aggregation failure is due to the existence of exogenous transaction costs. When the profit from trade is smaller than the transaction cost, investors stop trading and this prevents the complete learning of market fundamental. Décamps and Lovo (2002) and Cipriani and Guarino (2003) show that in a model where traders strategies are restricted (buy one lot, sell one lot, no trade) herd behavior and long run inefficiency can occur because of differences in agents' valuation for the asset.

In this paper we show that informational inefficiency is not necessarily linked to the presence of exogenous frictions in transaction prices due to inelastic prices (as in the rational herding literature), transaction cost (as in Lee (1998)) or exogenous difference in agents valuation for the object (as Cipriani and Guarino (2003)).

We analyze the effect that a change in the minimum trading unit (or lot size) ${ }^{3}$ has on informational inefficiency. On the one hand, an appropriate increase in the minimum trading unit can eliminate the long run mispricing. However, the choice of such an "informational-efficient lot size" is not robust to perturbations of the fundamentals of the economy. This suggests that it can be actually difficult to restore efficiency through the choice of an appropriate grid of tradable quantities. On the other hand, decreasing the lot size can reduce, but does not eliminate, the long term inefficiency. The latter observation allows to relate our analysis to the literature on stock splits. Starting from the observation that a stock split corresponds to a reduction of the minimum trading unit, we show that a stock splits reduce market inefficiency. Moreover, a stock split can temporarily restore the informativeness of trades and consequently increase price volatility. This could give reasons for the puzzling empirical findings that a stock split generates higher volatility in the stock's return (Ohlson and Penman's (1985), Koski (1998), Lamoureux and Poon (1987) and Amihud et al. (1999)).

\footnotetext{
${ }^{3}$ The minimum trading unit corresponds to the tick of the quantity grid.
} 
In Section 2 the notations, the assumptions and the basic structure of the model are presented. Section 3 shows the main result and analyzes the role of the minimum size of trade and stock splits. Section 4 extends the inefficiency result to a broader class of economies. In Section 5 we provide an example. Section 6 concludes. The proofs are in the Appendix.

\section{The model}

We consider a discrete time sequential trade model in the style of Glosten and Milgrom (1985) and Glosten (1989): a single risky asset is exchanged for money among market makers and traders. We denote with $\mathbf{v}=\mathbf{V}+\boldsymbol{\varepsilon}$ the (ex-post) liquidation value of one share of the risky asset. The random variable $\mathbf{v}$ is the sum of two components: a realized shock $\mathbf{V}$ on which agents are asymmetrically informed, and a noise $\boldsymbol{\varepsilon}$ that represents the shocks on fundamental whose realization is unknown to everybody such as for example future shocks ${ }^{4}$. For expositional clarity we introduce some simplifying assumptions on the distribution of $\mathbf{V}$ and $\boldsymbol{\varepsilon}$. The general case is discussed in Section 4. We assume that $\mathbf{V}$ and $\varepsilon$ are independently distributed and that $\mathbf{V}$ is equal to $\bar{V}$ with probability $\pi_{0}$ and to $\underline{V}<\bar{V}$ with probability $1-\pi_{0}$, moreover $\varepsilon$ has zero mean and strictly positive standard deviation $\sigma_{\varepsilon}$. Remark that $\mathbf{V}$ is an unbiased estimator of $\mathbf{v}$, but knowing $\mathbf{V}$ is not sufficient to know the exact value of $\mathbf{v}$.

There is a infinite countable set of traders, each one receives a private partially informative signal $\mathbf{s} \in\{l, h\}$. Signals are conditionally i.i.d. across traders and independent from $\varepsilon$ and from the compositions of traders' portfolios. Let $\operatorname{Pr}(\mathbf{s}=l \mid \mathbf{V}=\underline{V})=$ $\operatorname{Pr}(\mathbf{s}=h \mid \mathbf{V}=\bar{V})=p$ with $1 / 2<p<1$. The parameter $p$ represents the precision of the signal. Signal $l$ is more likely when $\mathbf{V}=\underline{V}$ and it can be interpreted as a "Bearish" signal. Similarly, $s=h$ can be interpreted as a "Bullish" signal. In other words, $\underline{V}<E[\mathbf{v} \mid s=l]<E[\mathbf{v}]<E[\mathbf{v} \mid s=h]<\bar{V}{ }^{5}$

\footnotetext{
${ }^{4}$ This way of modelling the information structure is borrowed from Biais, Martimort and Rochet
} (2000). The noise $\varepsilon$ takes into account that, as in reality, uncertainty is never completely resolved.

${ }^{5}$ The results of the paper do not rely on the independence between $\mathbf{V}$ and $\varepsilon$, their binomial distri- 
Trading mechanism. Trading occurs sequentially and time is discrete. Each time interval is long enough to accommodate the trade of at most one trader. At the beginning of each trading period a trader receives a private signal s and comes to the market with an endowment of shares known only to him. The trader submits a market order and market makers compete to fill the trader's order without knowing the trader's signal and portfolio composition. We assume that traders leave the market after they have had the opportunity to trade. We restrict the tradable quantities to belong to a quantity grid. We denote by $\delta$ the minimum trading unit. In other words, a trader's market order can be any integer multiple $Q$ (positive or negative) of a lot of $\delta$ shares of the asset. Our restriction to discrete quantities reflects the intrinsic nature of financial markets where only shares of a stock can be traded. If the exchange's rules allow to trade any integer number of shares, then $\delta=1$. In this case we say that trading mechanisms allows for odd-lot trading. By contrast, if $\delta$ is greater than 1, we say that the trading mechanisms is in round lots, and $\delta$ represents the amount of shares in a round lot. ${ }^{6}$

Market participants. Market makers are risk neutral and traders are risk averse. ${ }^{7}$ A trader's expected utility obtained from a portfolio that contains an amount $X$ of the risky assets and $M$ of cash is $E[u(M+X \mathbf{v})]$, where $u: \mathbb{R} \rightarrow \mathbb{R}, u^{\prime}>0$ and $u^{\prime \prime}<0$. For simplicity, we assume that all traders have the same utility function ${ }^{8}$ but they can differ for the initial compositions of their portfolios that are assumed to be independently and identically distributed. We denote $x$ and $m$ the initial amounts of risky asset and money respectively for a given trader. Note that $x$ is an integer number (positive or negative) as traders cannot hold fractions of shares of the asset, hence $x \in \mathbb{Z}$ and $m \in \mathbb{R}$. We will refer to $x$ as the trader's inventory. Consider a trader that reaches the market in a generic period $t$. For a set $\Theta \subset \mathbb{Z} \times \mathbb{R}$, we use $F(\Theta)=\operatorname{Pr}((x, m) \in \Theta)$ to denote the bution nor on the fact that the precision of the signal is the same for all the agents. See Section 4 for the treatment of the general case.

${ }^{6}$ Usually, a round lot consists of a lot of 100 shares or a multiple thereof.

${ }^{7}$ Though the crucial assumption is that market makers and traders have different degrees of risk aversion, the assumption that market makers are risk neutral simplifies the analysis.

${ }^{8}$ See Section 4 for the case of heterogenous traders. 
probability that the portfolio composition of this trader is in $\Theta$. We assume that there exist a bounded set $\widehat{\Theta} \subset \mathbb{Z} \times \mathbb{R}$ such that $F(\widehat{\Theta})=1$.

Public and private belief. We denote $H_{t}$ the history of trades (quantities and prices) up to time $t-1$. All the agents observe $H_{t}$ but they do not know the identity of past traders. As private signals provide information on the realization of $\mathbf{V}$ but not on the realization of $\varepsilon$, the learning process on the asset's fundamental only regards $\mathbf{V}$. The presence of $\varepsilon$ guarantees that the uncertainty on $\mathbf{v}$ remains even when the realization of $\mathbf{V}$ is commonly known. We denote $\pi_{t}=\operatorname{Pr}\left[\mathbf{V}=\bar{V} \mid H_{t}\right]$ the public belief at time $t$. If in period $t$ a trader submits an order of size $Q$, then public belief will evolve according to Bayes' rule: $\pi_{t+1}=\operatorname{Pr}\left[\mathbf{V}=\bar{V} \mid H_{t}, Q\right]$. We denote $v\left(\pi_{t}\right)=E\left[\mathbf{v} \mid H_{t}\right]=E\left[\mathbf{V} \mid H_{t}\right]$ the expectation of $\mathbf{v}$ when the belief is $\pi_{t}$. A trader refines public information with the one provided by his private signal. We denote $\pi_{t}^{s}=\operatorname{Pr}\left[\mathbf{V}=\bar{V} \mid H_{t}, s\right], s \in\{h, l\}$, an informed traders' belief at time $t$. With an abuse of notation we denote $v\left(\pi_{t}^{s}\right)=E\left[\mathbf{v} \mid H_{t}, s\right]$, $s \in\{h, l\}$.

Agents' behavior and equilibrium concept. At any given period $t$ a trader comes to the market, submits a market order and leaves the market. The trader expects a pricing schedule $P_{\delta}():. \mathbb{Z} \rightarrow \mathbb{R}$, with the interpretation that if he submits a market order $Q \in \mathbb{Z}$ positive (negative), then he will buy $\delta Q$ shares (resp. sell $\delta Q$ shares) and pay (resp. receive) $P_{\delta}(Q)$ per share. If a trader has a portfolio $(x, m)$, received the signal $s$ and expects a price schedule $P_{\delta}(Q)$, then he will demand the quantity

$$
Q^{*}\left(x, m, P_{\delta}, \pi_{t}, s\right)=\underset{Q \in \mathbb{Z}}{\arg \max } E\left[u\left(m+(x+\delta Q) \mathbf{v}-P_{\delta}(Q) \delta Q\right) \mid H_{t}, s\right] .
$$

Apart from the discreteness in the tradable quantities, competition among market makers is modeled as in Glosten (1989) or in Kyle (1985). Following these papers, as market makers are risk neutral and compete à la Bertrand to fill traders' orders, any trade of $\delta Q$ shares must lead to a zero conditional expected profit. Considering that market makers are ignorant of the portfolio composition and information of the trader 
who is trading, at any given period $t$ a price schedule must satisfy

$$
P_{\delta}(Q)=E\left[\mathbf{v} \mid H_{t}, Q^{*}=Q\right] .
$$

That is the market clearing price is equal to the market makers' expectation of $\mathbf{v}$ conditional on what they learn about $\mathbf{v}$ from the past and current trades. Thus, if period-t-trader receives signal $s$ and holds portfolio $(x, m)$, in equilibrium he will demand $Q^{*}\left(x, m, P_{\delta}, \pi_{t}, s\right)$ where the price schedule $P_{\delta}($.$) satisfies condition (1).$

\section{Informational inefficiency}

This section contains our main result showing that if: (i) traders are risk averse and market makers are risk neutral, (ii) agents can trade only discrete quantities; (iii) all the private information is not sufficient to completely resolve uncertainty, i.e. $\sigma_{\varepsilon}>0 ;{ }^{9}$ then in general the market is not informational efficient.

In the long run, the market is informational efficient if all the information dispersed among the traders in the economy is eventually incorporated into market prices. Considering that in our model, traders' private information only regards $\mathbf{V}, E[\varepsilon]=0$ and market makers are risk neutral, we have informational efficiency if the trading prices eventually converge to the realization of $\mathbf{V}$.

Definition 1: The market is strong-form informational efficient in the long run, if

$$
\lim _{t \rightarrow \infty} E\left[\left|P_{\delta}(Q)-\mathbf{V}\right|\right]=0
$$

where $P_{\delta}(Q)=E\left[\mathbf{V} \mid H_{t}, Q^{*}=Q\right]$.

Note that trading prices reflect the information content of past and current trades, and that the information content of a trader's order is bounded by the information content of the trader's private signal. As signals are not perfectly correlated with $\mathbf{V}$, in

\footnotetext{
${ }^{9}$ Consequently, uncertainty cannot be completely resolved even in the long term. We show however in Section 5 that this assumption is not necessary to generate inefficiency.
} 
order to achieve full efficiency, trades must never cease to be informative. We provide a formal definition of not informative trade:

Definition 2: A trader with portfolio $(x, m)$ who expects a price schedule $P_{\delta}$ is said to place a not informative order if the order is not affected by the trader's private signal, i.e.

$$
Q^{*}\left(x, m, P_{\delta}, \pi_{t}, h\right)=Q^{*}\left(x, m, P_{\delta}, \pi_{t}, l\right)
$$

According to this definition, a trader's order is not informative when even knowing the trader's portfolio composition $(x, m)$, the observation of his order does not allow to infer whether he received a bullish or a bearish signal. In other words, if a trade of size $Q$ is not informative, then $\operatorname{Pr}\left(Q^{*}=Q \mid \mathbf{V}=\bar{V}\right)=\operatorname{Pr}\left(Q^{*}=Q \mid \mathbf{V}=\underline{V}\right)$.

We will show that under some conditions on the distribution $F$, if in period $t$ the public belief $\pi_{t}$ is sufficiently close to 1 or to 0 , then the orders of all traders in the economy will not be informative. In this instance, the learning process stops and public belief and prices will not change anymore. Namely, trading prices will remain at level $P_{\delta}(Q)=E\left[\mathbf{V} \mid H_{t}\right]$ for all $Q$ and all following periods. This is usually referred as an informational cascade in the herding literature (see Bikchandani, Hirshleifer and Welch (1992)). Considering that no single order can fully reveal V, eventually belief $\pi_{t}$ will be close either to 1 or to 0 , and so an informational cascade will occur before market makers have completely learned V. Thus, contrary to the common wisdom, the trading prices cannot aggregate completely private information and the market is not informational efficient in the sense of Definition 1. This phenomenon can led to important long-run mispricing episodes. When, for instance, $\pi_{t}$ is sufficiently close to 1 but the actual fundamental $\mathbf{V}$ is equal to $\underline{V}$, the long term pricing error will be close to $v(\pi)-\underline{V} \simeq \bar{V}-\underline{V}$.

In order to understand why traders' orders eventually cease to be informative, it is useful to distinguish two components in the trading motivations of a risk averse agent: the inventory component and the information component. The inventory component reflects 
the agent's preference for low-risk-portfolios. It increases with the agent's degree of risk aversion, and the unresolved uncertainty about the asset's fundamental. The information component reflects the changes in traders' belief that follows a bearish or a bullish signal and can be measured by $\pi_{t}^{h}-\pi_{t^{\cdot}}^{l}{ }^{10}$ As signals are not perfectly informative about $\mathbf{V}$, the information component will decrease as the public belief $\pi_{t}$ approaches 0 or $1 .{ }^{11}$ In other words, if the trader is quite sure about the realization of $\mathbf{V}$, a partially informative private signal will affect his belief just slightly. Now, as a trader can demand only discrete quantities of the asset, a small change in his belief will in general not be sufficient to affect his demand ${ }^{12}$ and so we will have $Q^{*}\left(x, m, P_{\delta}, \pi_{t}, h\right)=Q^{*}\left(x, m, P_{\delta}, \pi_{t}, l\right)$. That means that, when the public belief is sufficiently close to 0 or to 1 , in general a trader's demand only reflects his inventory component.

The formal proof is slightly more complex. Indeed, it is always possible to imagine risk averse traders whose demand is informative no matter how close to 1 , or to 0 , is the public belief $\pi_{t}$. Thus, in order to characterize inefficient markets, we proceed as follows: firstly we identify the traders that submit informative orders even when $\pi_{t}$ is arbitrarily close to 1 or to 0 . Secondly, we show that the market is informational inefficient if the probability of observing such "informative traders" is zero.

Suppose that the belief $\pi_{t}$ is almost equal to 1 , or to 0 , and take a trader that before receiving the private signal, was indifferent between demanding an amount of $Q^{*}$ lots or $Q^{*}+1$. The demand of this trader will be informative. Indeed, after receiving a tiny informative signal this trader will demand $Q^{*}$ if the signal is bearish, whereas he will demand $Q^{*}+1$ if the signal is bullish. The following lemma characterizes the set of such traders:

Lemma 1 Take $\operatorname{Var}\left[\mathbf{V} \mid H_{t}\right]=0$. For any $n \in \mathbb{Z}$, there exist $x^{*}(n)$, with $\delta n<x^{*}(n)<$ $\delta(n+1)$, such that if a trader's inventory is $x^{*}(n)$, then trading $-n$ lots or $-n-1$ lots is optimal. If $\varepsilon$ is symmetrically distributed then $x^{*}(n)=\delta(n+1 / 2)$.

\footnotetext{
${ }^{10}$ Indeed, if signals are informative we have $\pi_{t}^{l}<\pi_{t}<\pi_{t}^{h}$.

${ }^{11}$ That is to say, $\lim _{\pi_{t} \rightarrow 0}\left(\pi_{t}^{h}-\pi_{t}^{l}\right)=0$ and $\lim _{\pi_{t} \rightarrow 1}\left(\pi_{t}^{h}-\pi_{t}^{l}\right)=0$.

${ }^{12}$ Note that this would not be the case if traders could demand a continuum of the asset.
} 
In other words, when $\pi_{t}$ is almost equal to 0 or to 1 , the only traders whose orders are informative, are those whose inventories are sufficiently close to $x^{*}(n)$ for some $n \in \mathbb{Z} .^{13}$

Consequently, if the quantity grid $\delta$ and -or- the traders' portfolio distribution $F$ are such that inventories of all traders are bounded away from $x^{*}(n)$ for all $n \in \mathbb{Z}$, then, by a continuity argument, when the public belief will be close enough to 0 or to 1 , the demand of all traders in the economy will reflect only the inventory component and will provide no information on $\mathbf{V}$. In this instance the flow of trade will be no more informative and long run inefficiency will occur. Now we turn to the formal statement of our result.

Proposition 1 Suppose that for all $n \in \mathbb{Z}$ the distribution of traders' portfolio composition $F$ is such that there is a zero probability that a trader's inventory is close to $x^{*}(n)$. Then there exists $\underline{\pi}>0$ and $\bar{\pi}<1$ such that if at time t the public belief $\pi_{t}<\underline{\pi}$ or $\pi_{t}>\bar{\pi}$, then in all trading periods $\tau \geq t$ the equilibrium is unique and such that:

(i) The price schedule satisfies $P_{\delta}(Q)=E\left[\mathbf{V} \mid H_{t}\right]$ for all $Q \in \mathbb{Z}$,

(ii) all traders's orders at date $\tau \geq t$ are not informative about $\mathbf{V}$.

As a consequence, $\pi_{\tau}=\pi_{t}$ for all $\tau>t$, full learning is impossible and trading prices cannot converge to the fundamental value of the asset.

Proposition 1 shows that, when the public belief $\pi_{t}$ is sufficiently large or sufficiently small (i.e., $\operatorname{Var}\left[\mathbf{V} \mid H_{t}\right]$ small) then the equilibrium exists, it is unique and noninformative. Precisely the equilibrium price schedule must be $P_{\delta}(Q)=v\left(\pi_{t}\right)$ for all tradable quantities $Q \in \mathbb{Z}$. The result is fairly robust as it is obtained without specifying the traders' utility function nor the precise distributions of $\varepsilon$. Note that in these types of models, existence of equilibrium is an issue. For instance Glosten (1989) proves the existence and uniqueness of equilibrium for a particular range of the parameters ${ }^{14}$

\footnotetext{
${ }^{13}$ Note that even when $\pi_{t}=1$ or $\pi_{t}=0$ the asset is still risky because of the $\varepsilon$ component. Thus traders trade in order to hedge the risk of their portfolio.

${ }^{14}$ In short, the variance of the fundamental value and the precision of private signals must be sufficiently small.
} 
with the further restriction of CARA utility function and normal distributions.

When the hypothesis of Proposition 1 are satisfied, the financial market cannot be informational efficient as the learning process stops as soon as the public belief $\pi_{t}$ crosses one of the threshold $\underline{\pi}$ or $\bar{\pi}$. We call the regions $(0, \underline{\pi})$ and $(\bar{\pi}, 1)$ information traps. Indeed if after a trading history the public belief $\pi_{t}$ belongs to one of these two regions, it will not move anymore. In this case, all quantities of the asset will be traded at $v\left(\pi_{t}\right)$ per share, and the trading price will not change for all the subsequent periods $\tau>t$. This can potentially lead to highly inefficient markets. Namely, if $\mathbf{V}=\underline{V}$ and $\pi_{t} \in(\bar{\pi}, 1)$, then no matter the trading history observed after $t$, prices will remain at level $P(Q)=v\left(\pi_{t}\right)$ much larger than $\underline{V}$.

\subsection{Rounds lots, odd lots and stock splits}

One of the crucial factors that impedes the aggregation of information is the restriction of trades to integer multiples of a minimum amount of shares $\delta$. The minimum unit of trade is chosen by the exchange regulator but it can also be indirectly affected by the decision of the issuer of the equity to split its stock. This section studies how the size of the minimum unit of trade affects the informational efficiency properties of the market.

The following two corollaries enlighten the role of the lot size $\delta$ in exacerbating and mitigating informational inefficiency. Surprisingly, it turns out that it could be optimal to increase $\delta$ in order to restore the market informational efficiency. Precisely, Corollary 1 states that if the quantity grid is the finest one, that is $\delta=1$, then long run informational inefficiency occurs almost surely for all discrete distribution $F$ of the traders' portfolios. Corollary 2 shows that when the noise $\varepsilon$ is symmetrically distributed it is possible to find a minimum unit of trade that guarantees long run informational efficiency.

Corollary 1 An odd-lot trading mechanism is informational inefficient.

Corollary 2 If $\varepsilon$ is symmetrically distributed, then in a round-lot mechanism, long run informational efficiency can be obtained only by choosing a minimum trading unit $\delta$ such 
that

$$
\sum_{n \in \mathbb{Z}} F\left(\left\{x=\delta\left(n+\frac{1}{2}\right)\right\}\right)>0 .
$$

In order to have informational efficiency in a round lot mechanism, the size of the round lot $\delta$ must be chosen so that the probability of observing informative orders is positive also when the public belief $\pi_{t}$ reaches extreme levels. Thus, an exchange regulator that is mainly concerned with the problem of informational efficiency could choose the minimum trading unit that maximizes the probability of observing orders from traders whose inventory is $x^{*}(n)$. For Lemma 1 , when $\varepsilon$ is symmetrically distributed, we have $x^{*}(n)=\delta(n+1 / 2)$ that implies that the optimal $\delta$ only depends on the distribution function $F$ and not on traders' utility functions. However, it is worth stressing that a mean-preserving asymmetric perturbation of the distribution of $\varepsilon$ would change the value $x^{*}(n)$ and this would restore informational inefficiency in the economy. Roughly speaking, informational efficiency appears to be very fragile.

In addition to the exchange's regulation on the size of tradable lots, the minimum unit of trade of an equity can be affected by the company decision to split its stock. With a stock split a company entitles its share holders with $N>1$ new shares for each old share. In other words a stock split increases the number of shares outstanding without increasing the company's capital and without affecting the ownership structure. As the market value of a company is independent from the number of shares outstanding, in fact, a stock split corresponds to a reduction of the minimum unit of trade. If before the stock split the fundamental value of one unit of trade was $\delta \mathbf{v}$, after splitting each share into $N$ new shares, the fundamental value of each new share will be $\mathbf{v}^{\prime}=\mathbf{v} / N$. Therefore, the value of one lot of $\delta$ new shares is $\delta \mathbf{v}^{\prime}=\frac{\delta}{N} \mathbf{v}$. This is perfectly equivalent to reducing the minimum unit of trade from $\delta$ shares to $\delta / N$ shares without splitting the stock.

The following corollary shows that a stock split shrinks the information traps and consequently reduces the informational inefficiency of the market. More formally let $(0, \underline{\pi})$ and $(\bar{\pi}, 1)$ be the information traps for a stock traded in odd lots. Denote by 
$(0, \underline{\pi}(N))$ and $(\bar{\pi}(N), 1)$ the new information traps after splitting each share into $N$ new shares, then we have

Corollary 3 Consider an odd lots trading mechanism and suppose that each share of the stock is split into a finite number $N$ of new shares. If $N$ is sufficiently large, then $0<\underline{\pi}(N)<\underline{\pi}$ and $\bar{\pi}<\bar{\pi}(N)<1$.

This result helps understanding the "stock split puzzle": if the market value of a firm's equity is independent from the number of shares outstanding, a stock splits should not affect the distribution of stocks returns. However, several empirical studies on stock splits (Ohlson and Penman (1985) and Koski (1998)) find that stock return volatility increases after a stock splits. How is Corollary 3 related to price volatility? First, note that as long as $\pi_{t}$ does not lie into an information trap, trades are informative and trading prices can vary within a range of about $v(\bar{\pi})-v(\underline{\pi})$. Corollary 3 shows that a stock split increases this range and this allows a higher volatility for prices. Second, a stock split can increase the price volatility by restoring the informativeness of trade in case an informational cascade is happening. For example, suppose that before the split, the public belief $\pi_{t}$ was in an information trap. Then the asset is traded only for inventory reasons, trades do not transmit information on the asset fundamental, and trading prices will be steady. In case of a stock split, the information traps shrink, and for the same level of public belief $\pi_{t}$, informativeness of trade can be temporarily restored. Thus the volatility of trading prices increases. ${ }^{15}$ Finally, suppose that traders differ in their degree of risk aversion, it can be shown that for some $\pi_{t}$ close to 1 or to 0 , traders that are sufficiently risk averse will trade mainly for hedging and will submit orders that are less informative in comparison to those coming from less risk averse traders. After a stock split even very risk averse traders can decide to speculate on their information as this will require a smaller investment in the stock. As a consequence the proportion of

\footnotetext{
${ }^{15}$ Besides, the same mechanism can induce managers with favorable information about their companies to split their share in order to allow a positive reaction of prices to the order flow. This would provide a further explanation to the empirical observation that stock splits lead to higher stock prices as shown by Lamoureux and Poon (1987) and Amihud et al. (1999).
} 
informative trade increases, the price sensitivity to orders increases generating a larger price volatility.

\section{A general model}

In order to simplify the analysis, in the previous section we assumed homogeneity of traders' utility functions, binomial distribution for $\mathbf{V}$ and $\mathbf{s}$, and independence between $\mathbf{V}$ and $\varepsilon$. In this section we discuss the robustness of our result when these three assumptions are relaxed. We denote by $\mathbf{v}(\mathbf{Z}, \mathbf{N})$ the fundamental value of the asset that will depend on two components: a realized shock $\mathbf{Z}$ on which agents are asymmetrically informed, and a noise $\mathbf{N}$ that represents the shocks on fundamentals whose realization is unknown to everybody. Random variables $\mathbf{Z}$ and $\mathbf{N}$ may lay in any measurable space, whereas $\mathbf{v}$ takes value in $\mathbb{R}^{+}$. We assume that $\mathbf{Z}$ is a discrete random variable and that the aggregation of all the private information that is dispersed among investors allows to know its realization. Still, knowing $\mathbf{Z}$ will not be sufficient to completely resolve the uncertainty on the fundamental value of the asset because of $\mathbf{N}$. We denote $\mathbf{V}=E[\mathbf{v} \mid \mathbf{Z}]$ the expected fundamental value of the asset after aggregating all the private information. We assume that $\mathbf{V}$ is a discrete random variable that takes value in a compact set $\Omega \in \mathbb{R}^{+}$. Let $\boldsymbol{\varepsilon}=\mathbf{v}-\mathbf{V}$ be the remaining error. The random variable $\boldsymbol{\varepsilon}$ has zero mean and positive standard deviation $\sigma_{\varepsilon}>0$. Thus, we can assume without loss of generality that $\mathbf{v}=\mathbf{V}+\boldsymbol{\varepsilon}$ and that agents private information regards $\mathbf{V}$ but not $\boldsymbol{\varepsilon}$. Note that the random variables $\mathbf{V}$ and $\varepsilon$ are not necessarily independently distributed. Still $E[\mathbf{V}]$ is an unbiased estimator of $\mathbf{v}: E[\varepsilon]=0$ and $\sigma_{\varepsilon}>0$.

Each trader receives a partially informative private signal $\mathbf{s}$ that takes value in a discrete compact set $\Sigma$. Without loss of generality, we assume that conditional on the realization of $\mathbf{V}$, private signals are independent. We assume that for all $V \in \Omega$ and $s \in \Sigma$, we have $\operatorname{Pr}(\mathbf{s}=s \mid \mathbf{V}=V)>0$. That means that private signals are not perfectly informative as each realization of the signal is compatible with all realizations of $\mathbf{V} .{ }^{16}$

\footnotetext{
${ }^{16}$ This condition is equivalent the condition $p_{q l}>0$ at page 1000 in Bikhchandani et al. (1992).
} 
Traders are risk averse in the sense that each trader's utility function is increasing, twice differentiable and strictly concave. Apart from this assumption, traders can differ in their utility functions and in the composition of their portfolio that does not depends on the private signal. In other words, for all levels of inventory $x$ and $x^{\prime}$ we have

$$
E[\mathbf{V} \mid \text { a trader's inventory is } x]=E\left[\mathbf{V} \mid \text { a trader's inventory is } x^{\prime}\right] \text {. }
$$

This last assumption guarantees that whenever a trader exchanges only for inventory reason his order will provide no additional information about $\mathbf{V}$.

Note that, similarly to the simpler set-up of the previous section, we have market informational efficiency when $\lim _{t \rightarrow \infty} E\left[\left|P_{\delta}(Q)-\mathbf{V}\right|\right]=0$, where $P_{\delta}(Q)=E\left[V \mid H_{t}, Q=Q^{*}\right]$. Consequently, market informational efficiency requires $\lim _{t \rightarrow \infty} \operatorname{Var}\left[\mathbf{V} \mid H_{t}\right]=0$.

It is possible to extend all results of Section 3 to this general framework. For expositional simplicity we only focus the informational inefficiency of odd lot trading mechanisms.

Proposition 2 In an odd-lot trading mechanisms $(\delta=1)$, there exist $\varsigma>0$ such that if $\operatorname{Var}\left(\mathbf{V} \mid H_{t}\right)<\varsigma$, then in all trading periods $\tau \geq t$ the equilibrium is unique and such that:

(i) The price schedule satisfies $P_{1}(Q)=E\left[\mathbf{V} \mid H_{t}\right]$ for all $Q \in \mathbb{Z}$.

(ii) A trader with portfolio $x$ will trade exactly $-x$ no matter the signal he received.

As a consequence, full learning is impossible and trading prices cannot converge to the fundamental value of the asset.

Proposition 2 shows that market inefficiency does not rely on the simplifying assumptions we have introduced in the previous sections. Indeed, even the less risk averse trader who received the most precise signal, will eventually trade only for inventory reason once the public beliefs are sufficiently precise about market fundamentals, i.e. once $\operatorname{Var}\left[\mathbf{V} \mid H_{t}\right]$ is sufficiently small. Thus, informational inefficiency arises when there is a general agreement on the asset's fundamental. In these cases, informed traders are prone to ignore their signals and trade only for inventory reasons. The more interesting point 
is that learning can stop when $\operatorname{Var}\left[\mathbf{V} \mid H_{t}\right]$ is small but the market belief are wrong, in the sense that the actual realization of $\mathbf{V}$ is bounded away from $E\left[\mathbf{V} \mid H_{t}\right]$.

Note also that our result is obtained assuming that there is a zero measure of risk neutral traders. Décamps and Lovo (2002) in a simplified model show that long term mispricing can also occur when traders are risk neutral provided that dealers are risk averse. This suggest that what lead to inefficiency is not the absence of risk neutral traders but the absence of traders whose utility functions are identical to those of market makers.

\section{An example}

Definition 1 and equation (1) suggest that informational efficiency properties of the market can be measured by the random variable $L T P E=\lim _{t \rightarrow \infty}\left|\mathbf{V}-E\left[\mathbf{V} \mid H_{t}\right]\right|$. For Proposition 1, as soon as the public belief $\pi$ reaches one of the two information traps, we have $P_{\delta}(Q)=v(\pi)$ for all $Q$. Therefore, in the long run trading price will be either close to $v(\underline{\pi})$ or to $v(\bar{\pi})$. Thus, a threshold $\underline{\pi}$ close to 0 and a threshold $\bar{\pi}$ close to 1 correspond to a relatively efficient market. Indeed, on the one hand the prices can reach a region that is relatively close to the true value of $\mathbf{V}$ and, on the other hand, the probability of observing a trading history that lead the public belief into the "wrong" information trap is low.

How is informational inefficiency affected by the precision of private signals $p$, the traders' degree of risk aversion and by the proportion of the fundamental volatility that can be explained with private information? In order to answer these questions we study a specification of our model of Section 3. Namely, we consider an odd-lot trading mechanism $(\delta=1)$, and we assume that traders have negative exponential utility function (with risk aversion coefficient $\gamma$ ) and that $\varepsilon$ is normally distributed. ${ }^{17}$ Then we study how $\underline{\pi}, \bar{\pi}$ and LTPE are affected by traders' $p, \gamma$ and $\sigma_{\varepsilon}$.

From Corollary 1, we already know that such a market cannot be informational

\footnotetext{
${ }^{17}$ These are the standard assumptions used in financial microstructure theory.
} 
efficient. The following lemma allows us to characterize the thresholds $\underline{\pi}$ and $\bar{\pi}$ and to perform some comparative statics.

Lemma 2 Let $u(W)=-e^{-\gamma W}$, let $\varepsilon \hookrightarrow N\left(0, \sigma_{\varepsilon}\right)$ and let $\delta=1$, then $\bar{\pi}$ (resp. $\underline{\pi}$ ) is the minimum $\pi>1 / 2$ (resp. maximum $\pi<1 / 2$ ) such that the following two inequalities are satisfied

$$
\begin{gathered}
e^{-\gamma\left(v(\pi)+\gamma \sigma_{\varepsilon}^{2} / 2\right)} \leq \pi^{h} e^{-\gamma \bar{V}}+\left(1-\pi^{h}\right) e^{-\gamma \underline{V}}, \\
e^{\gamma\left(v(\pi)-\gamma \sigma_{\varepsilon}^{2} / 2\right)} \leq \pi^{l} e^{\gamma \bar{V}}+\left(1-\pi^{l}\right) e^{\gamma \underline{V}} .
\end{gathered}
$$

where $\pi^{h}=\frac{\pi p}{\pi p+(1-\pi)(1-p)}$ and $\pi^{l}=\frac{(1-\pi) p}{\pi(1-p)+(1-\pi) p}$.

Note first that if $\gamma$ is sufficiently large, then inequalities (3) and (4) will be met for all $\pi \in[0,1]^{18}$. This happens because when traders are sufficiently risk averse the informational content of their order vanishes as they mainly trade to reduce the risk of their portfolio.

Similarly, when the precision of private signals is small ( $p$ is close to $1 / 2), \pi^{h}$ is close to $\pi^{l}$ and inequalities (3) and (4) will be satisfied even if $\sigma_{\varepsilon}$ is arbitrarily small. ${ }^{19}$ This implies that the presence of the additional noise $\varepsilon$ is not a necessary condition to obtain informational inefficiency. Thus, even if the aggregation of all private information could resolve uncertainty almost completely, when traders' information is not precise, the existence of a minimum trading size will induce traders to neglect their information and this will impede the convergence of prices to fundamental.

Finally, remark that there exists $\sigma_{\varepsilon}$ sufficiently large such that no matter the level of public belief or the information content of the private signal, the two inequalities are satisfied. This means that if the uncertainty coming from the noise $\varepsilon$ is sufficiently large with respect to the information provided by the component $\mathbf{V}$, then even signals that are perfectly informative about $\mathbf{V}$ will not be reflected in traders' orders. Indeed, the

\footnotetext{
${ }^{18}$ Indeed, an increase in $\gamma$ increases the convexity of the exponential. Moreover, a sufficiently large increase in $\gamma$ reduces the left hand sides of expressions (3) and (4).

${ }^{19}$ This follows from the convexity of the exponential.
} 
asset will be too risky to be hold even by traders that are perfectly informed about one component of the asset fundamental value.

To sum up, when i) the traders' risk aversion is high; or ii) the precision of private signals is low; or iii) the volatility in market fundamental is mostly due to shocks on which there is no information, then even an infinite sequence of trades will not allow the market to aggregate the relevant private information dispersed among traders.

We conclude with a numerical example that illustrates our result. Take a risky asset whose ex-ante expected fundamental value is $E[\mathbf{v}]=35 \$$, and whose ex-ante standard

deviation is $\sigma=\sqrt{\sigma_{\mathbf{V}}^{2}+\sigma_{\varepsilon}^{2}}=7.5 \$$ that is $25 \%$ of its ex-ante value, where $\sigma_{\mathbf{V}}^{2}=\frac{1}{4}(\bar{V}-$ $\underline{V})^{2}$. This corresponds to the magnitude of the average share price and annual volatility in the New York Stock Exchange. And suppose that $\sigma_{V} / \sigma=0.1$, that means that $10 \%$ of the annual volatility of $\mathbf{v}$ measured by $\sigma$ could be explained with all the private information available in a given period, say one trading week. Then for a level of risk aversion $\gamma=0.001$ it results $\bar{\pi}=0.983$ and $\underline{\pi}=0.017$, that corresponds to a minimum LTPE of $0.03 \$$ and a maximum LTPE of $1.72 \$$ that is $4.85 \%$ of the ex-ante value of the asset.

\section{Conclusion}

We studied the informational efficiency properties of a financial market when one takes into account two factors: first, agents can trade only integer quantities of the asset; second, traders and market makers do not have the same degree of risk aversion. We show that in general market fails to fully aggregate relevant private information. In other words the long run trading prices are bounded away from the value of the asset given all the information dispersed in the market. Indeed, when public belief are sufficiently precise, the equilibrium is unique and such that traders' orders provide no information about the asset's fundamental value.

We show that an appropriate increase of the minimum trading unit can restore completely long run informational efficiency. Still, the choice of an "efficient quantity 
grid" is not robust to small perturbation of the fundamentals' distribution. We show that decreasing the minimum unit of trade can reduce but does not eliminate market inefficiency. This provides an alternative explanation of the empirical observation that stock splits increase stock return volatility.

In our set-up, information aggregation failure potentially leads to large long-run pricing errors. With an example, we show that market inefficiency increases with traders' risk aversion and with the proportion of fundamental's volatility that cannot be explained with private information and it decreases with the precision of informed traders' signals. It would be interesting to obtain experimental evidence of these predictions.

The fact that our results are obtained within a fairly general framework and by introducing reasonable assumptions into standard microstructure models, suggests that the informational efficiency hypothesis is not necessarily compatible with the way financial economists are used to model the trading process in financial markets.

\section{Appendix}

Proof of Lemma 1: Note that $\operatorname{Var}\left[V \mid H_{t}\right]=0$ implies either $\pi_{t}=1$ or $\pi_{t}=0$. Take $\pi_{t}=1$, in this case $P_{\delta}(Q)=E\left[\mathbf{V} \mid H_{t}, Q\right]=\bar{V}$. Let $U(x, Q)$ be a traders' expected utility from trading $Q$ at price $\bar{V}$ when his initial inventory is $x$, i.e. $U(x, Q)=E[u(m+x \bar{V}+$ $(x+\delta Q) \varepsilon)]$. Then from risk aversion and from the fact that traders wealth is bounded, we have that $U(\delta n,-n)>U(\delta n,-(n+1))$ and $U(\delta(n+1),-n)<U(\delta(n+1),-(n+1))$. Thus, from the continuity of $U$ in $x$ there exists $x^{*}(n) \in(\delta n, \delta(n+1))$ such that if $x=x^{*}(n)$, then the trader is indifferent between trading $-n$ lots or $-(n+1)$ lots.

In order to see that when $x=x^{*}(n)$ both these quantities are optimal, note that if

the trader could trade a continuum of quantities, then he would trade exactly $-\frac{x}{\delta}$. The trader is however constrained to trade integer multiples of $\delta$. Taking advantage from the concavity in $Q$ of $U(x, Q)$, the constrained optimal tradable quantities are the closest to $-\frac{x}{\delta}$, that are $-\delta n$ and $-\delta(n+1)$. Finally if $\varepsilon$ is symmetrically distributed, then $\varepsilon$ and $-\varepsilon$ are identically distributed and so $U(\delta(n+1 / 2),-n)=U(\delta(n+1 / 2),-(n+1))$ that 
means $x^{*}(n)=\delta(n+1 / 2)$. The proof for the case $\pi_{t}=0$ is symmetric.

Proof of Proposition 1: Note first that as quotes must satisfy equation (1) and the informativeness of an order is bounded by the precision of a traders' signal, we have that in equilibrium, at any date $t, v\left(\pi_{t}^{l}\right) \leq P_{\delta}(Q) \leq v\left(\pi_{t}^{h}\right)$ for all $Q \in \mathbb{Z}$. Thus, for any price schedule $P_{\delta}(Q)$ satisfying this property and for a trader with portfolio $(x, m)$ we have:

$$
\begin{aligned}
E\left[u\left(m+(x+\delta Q) \mathbf{v}-\delta Q v\left(\pi_{t}^{h}\right)\right) \mid H_{t}, s\right] & \leq E\left[u\left(m+(x+\delta Q) \mathbf{v}-\delta Q P_{\delta}(Q)\right) \mid H_{t}, s\right] \leq \\
& \leq E\left[u\left(m+(x+\delta Q) \mathbf{v}-\delta Q v\left(\pi_{t}^{l}\right)\right) \mid H_{t}, s\right]
\end{aligned}
$$

for $Q$ positive, and

$$
\begin{aligned}
E\left[u\left(m+(x+\delta Q) \mathbf{v}-\delta Q v\left(\pi_{t}^{l}\right)\right) \mid H_{t}, s\right] & \leq E\left[u\left(m+(x+\delta Q) \mathbf{v}-\delta Q P_{\delta}(Q)\right) \mid H_{t}, s\right] \leq \\
& \leq E\left[u\left(m+(x+\delta Q) \mathbf{v}-\delta Q v\left(\pi_{t}^{h}\right)\right) \mid H_{t}, s\right]
\end{aligned}
$$

for $Q$ negative.

Note that $\pi_{t}^{s}$ is continuous in $\pi_{t}$ and that $u$ is a continuous function. Moreover, when $\pi_{t}$ is close to 1 or to 0 , an informative signal affects slightly the informed trader belief, indeed $\pi_{t}^{l}<\pi<\pi_{t}^{h}$ and $\lim _{\pi_{t \rightarrow 1}}\left(\pi_{t}^{h}-\pi_{t}^{l}\right)=\lim _{\pi_{t \rightarrow 0}}\left(\pi_{t}^{h}-\pi_{t}^{l}\right)=0$. Thus, we have that:

$$
\begin{aligned}
\lim _{\pi_{t \rightarrow 1}} E\left[u\left(m+(x+\delta Q) \mathbf{v}-\delta Q v\left(\pi_{t}^{h}\right)\right) \mid s\right] & =\lim _{\pi_{t \rightarrow 1}} E\left[u\left(m+(x+\delta Q) \mathbf{v}-\delta Q v\left(\pi_{t}^{l}\right)\right) \mid s\right] \\
& =E[u(m+x \bar{V}+(x+\delta Q) \boldsymbol{\varepsilon})]
\end{aligned}
$$

From Lemma 1 we know that if $x \neq x^{*}(n)$ for all $n \in \mathbb{Z}$, then there exist a unique $\widehat{Q}$, such that for all $Q \neq \widehat{Q}$, we have

$$
E[u(m+x \bar{V}+(x+\delta \widehat{Q}) \varepsilon))]>E[u(m+x \bar{V}+(x+\delta Q) \varepsilon)]
$$


Thus, from expression (7), it must be that for $\pi_{t}$ sufficiently close to 1 and for all $Q \neq \widehat{Q}$

$$
\begin{aligned}
& E\left[u\left(m+(x+\delta \widehat{Q}) \mathbf{v}-\delta \widehat{Q} v\left(\pi_{t}^{h}\right)\right) \mid H_{t}, s\right]>E\left[u\left(m+(x+\delta Q) \mathbf{v}-\delta Q v\left(\pi_{t}^{l}\right)\right) \mid H_{t}, s\right] \\
& E\left[u\left(m+(x+\delta \widehat{Q}) \mathbf{v}-\delta \widehat{Q} v\left(\pi_{t}^{l}\right)\right) \mid H_{t}, s\right]>E\left[u\left(m+(x+\delta Q) \mathbf{v}-\delta Q v\left(\pi_{t}^{h}\right)\right) \mid H_{t}, s\right] .
\end{aligned}
$$

Now take an informed trader whose inventory $x$ is bounded away from $x^{*}(n)$ for all $n \in \mathbb{Z}$, and suppose he expects a price schedule $P_{\delta}(Q)$. His maximization problem will be:

$$
\underset{Q \in \mathbb{Z}}{\arg \max } E\left[u\left(m+(x+\delta Q) \mathbf{v}-\delta Q P_{\delta}(Q)\right) \mid H_{t}, s\right] .
$$

Then expressions (5), (6), (8) and (9) imply

$$
E\left[u\left(m+(x+\delta \widehat{Q}) \mathbf{v}-\delta \widehat{Q} P_{\delta}(\widehat{Q})\right) \mid H_{t}, s\right]>E\left[u\left(m+(x+\delta Q) \mathbf{v}-\delta Q P_{\delta}(Q)\right) \mid H_{t}, s\right]
$$

for all $Q \neq \widehat{Q}$. That is if $\pi_{t}$ is sufficiently close to 1 , this trader will trade a quantity $\widehat{Q}$ no matter he received a bearish or a bullish signal. Therefore his action will provide no information on $\mathbf{V}$. To conclude the proof it is sufficient to observe that because of the hypothesis on $F$, for all $n \in \mathbb{Z}$ there is no trader whose inventory is not bounded away from $x^{*}(n)$. Thus, traders' demand is not informative, and so, from equation (1), the price schedule must be $P_{\delta}(Q)=E\left[\mathbf{v} \mid H_{t}\right]$ for all $Q \in \mathbb{Z}$. In order words, when $\pi_{t}$ is sufficiently close to 1 , there exist no equilibrium where the traders orders are informative. In order to prove that a not informative equilibrium exist, it is sufficient to observe that for $\pi_{t}$ close to $1, Q^{*}\left(x, m, v\left(\pi_{t}\right), \pi_{t}, s\right)=\widehat{Q}$ for all $s$. An identical argument applies for $\pi_{t}$ sufficiently close to 0 .

Proof of Corollary 1: Simply remark that from Lemma 1, when $\delta=1$ we have $x^{*}(n) \in(n, n+1)$. That means that when $\pi_{t}$ reaches extreme levels, the only traders whose orders are informative are traders that hold fractions of the asset. ${ }^{20}$ However, all

\footnotetext{
${ }^{20}$ Moreover, as the traders wealth is bounded, we know that $x^{*}(n)$ is bounded away from $n$ or $n+1$.
} 
traders in the economy hold only integer amounts of the asset, $x \in \mathbb{Z}$, and thus, from Proposition 1, eventually trade will stop providing information on $\mathbf{V}$.

Proof of Corollary 2: From Lemma 1 and Proposition 1 we know that the only traders whose orders are informative even when $\pi_{t}$ is arbitrarily close to 0 or to 1 are those whose inventory is equal to $x^{*}(n)$ for some $n \in \mathbb{Z}$. Moreover, if $\varepsilon$ is symmetrically distributed we know that $x^{*}(n)=\delta\left(n+\frac{1}{2}\right)$. Therefore $\delta$ should be chosen such that there exist a positive probability of observing these traders, thus the inequality (2).

Proof of Corollary 3: Notice first that Corollary 1 implies that after splitting each old share into a finite number $N$ of new shares, there still exist information traps $(0, \underline{\pi}(N))$ and $(\bar{\pi}(N), 1)$.

Let $\pi_{t} \in(0,1)$ be the public belief at time $t$. In the following we denote $q^{*}(s)$ the demand of an hypothetical trader that received signal $s$ and that can trade any real amount of the asset. That is to say, if his portfolio is $(x, m)$, we have

$$
q^{*}(s)=\arg \max _{q \in \mathbb{R}} E\left[u\left(m+(x+q) \mathbf{v}-q v\left(\pi_{t}\right)\right) \mid H_{t}, s\right]
$$

Note that as our hypothetical trader can trade any real quantity and signals are informative, we have $\eta=\left|q^{*}(h)-q^{*}(l)\right|>0$. Without loss of generality we assume thereafter $q^{*}(h)>q^{*}(l)$.

Consider now the situation where trading is discrete and suppose that at time $t$, before the stock split, it results $\bar{\pi} \leq \pi_{t}<1$, i.e., trades are not informative. We prove by contradiction that there is finite integer $N$ such that after splitting each share into $N$ new shares the information trap shrinks, i.e., $\bar{\pi}(N)>\bar{\pi}$. Take $N \geq 2 / \eta$, and suppose that after the stock split trades are still not informative. In this case $P(Q)=v\left(\pi_{t}\right)$ for all $Q$, and the trader maximization problem in odd lots can be written as follows

$$
\max _{Q \in \Gamma} E\left[u\left(m+(x+Q) \mathbf{v}-Q v\left(\pi_{t}\right)\right) \mid H_{t}, s\right]
$$

where $\Gamma=\left\{\ldots-\frac{2}{N},-\frac{1}{N}, 0, \frac{1}{N}, \ldots, \frac{n}{N} \ldots\right\}$. From $N \geq 2 / \eta$ it follows that there exist at least two tradable quantities $\left\{Q^{\prime}, Q^{\prime \prime}\right\} \in \Gamma$ such that $q^{*}(l) \leq Q^{\prime}<Q^{\prime \prime} \leq q^{*}(h)$. As 
$E\left[u\left(m+(x+q) \mathbf{v}-q v\left(\pi_{t}\right)\right) \mid H_{t}, s\right]$ reaches its maximum in $q^{*}(s)$ and is strictly concave in $q$, it must result that

$$
\begin{aligned}
\underset{Q \in \Gamma}{\arg \max } E\left[u\left(m+(x+Q) \mathbf{v}-Q v\left(\pi_{t}\right)\right) \mid H_{t}, l\right] \leq Q^{\prime}<Q^{\prime \prime} & \\
& \underset{Q \in \Gamma}{\arg \max } E\left[u\left(m+(x+Q) \mathbf{v}-Q v\left(\pi_{t}\right)\right) \mid H_{t}, h\right] .
\end{aligned}
$$

That implies that the trader demand is informative, thus a contradiction. The proof for $\underline{\pi}$ is symmetric.

Proof of Proposition 2: The proof is similar to the proof of Proposition 1. First we show that the price schedule has an upper and a lower bound that converge to $E\left[\mathbf{V} \mid H_{t}\right]$ as public information becomes sufficiently precise. Then we show that, whenever trading prices are sufficiently close to $E\left[\mathbf{V} \mid H_{t}\right]$ and public information is sufficiently precise, all risk averse traders order's will not be affected by their private signals. Thus, the informational content of the order flow vanishes and prices will not converge to fundamental.

We say that the public belief is sufficiently precise at time $t$ if $\operatorname{Var}\left(\mathbf{V} \mid H_{t}\right)$ is positive but sufficiently close to 0 . Remark that preciseness of public belief has nothing to do with the fact that agents' belief are actually correct. Indeed, very precise public belief can turn out to be completely wrong.

Note that, from standard property of the conditional variance we have $\operatorname{Var}\left(\mathbf{V} \mid H_{t}\right)=$ $E\left[\operatorname{Var}\left(\mathbf{V} \mid H_{t}, \mathbf{s}\right)\right]+\operatorname{Var}\left(E\left[\mathbf{V} \mid H_{t}, \mathbf{s}\right]\right)$. Thus, if $\operatorname{Var}\left(\mathbf{V} \mid H_{t}\right)<\varsigma$, then it is also the case that $\operatorname{Var}\left(\mathbf{V} \mid H_{t}, \mathbf{s}\right)<\varsigma$. Moreover, from $\operatorname{Pr}(\mathbf{s}=s \mid \mathbf{V}=V)>0$ for all $V \in \Omega$ and $s \in \Sigma$ and from the compactness of $\Omega$ and $\Sigma$, we have that for any couple of signals $s$ and $s^{\prime}$. It results

$$
\begin{aligned}
\lim _{\varsigma \rightarrow 0} \max _{V \in \Omega} \mid & \operatorname{Pr}\left(\mathbf{V}=V \mid H_{t}, s\right)-\operatorname{Pr}\left(\mathbf{V}=V \mid H_{t}, s^{\prime}\right) \mid=0, \\
& \operatorname{Pr}\left(\mathbf{V}=V \mid H_{t}, s\right)<\frac{\varsigma}{\left(V-E\left[\mathbf{V} \mid H_{t}, s\right]\right)^{2}} \quad \forall V \in \Omega .
\end{aligned}
$$

In other words, if the public belief is sufficiently precise, then public information overwhelms private information and private signals will affect private beliefs just slightly. 
Now, for any finite history $H_{t}$ it is always possible to find two signals in $\Sigma$, that with an abuse of notation we will denote $l$ and $h$, such that

$$
E\left[\mathbf{V} \mid H_{t}, \mathbf{s}=l\right] \leq E\left[\mathbf{V} \mid H_{t}, \mathbf{s}=s\right] \leq E\left[\mathbf{V} \mid H_{t}, \mathbf{s}=h\right], \quad \forall s \in \Sigma
$$

Expression (10) implies that $E\left[\mathbf{V} \mid H_{t}, \mathbf{s}=h\right]-E\left[\mathbf{V} \mid H_{t}, \mathbf{s}=l\right]$ is close to 0 as $\operatorname{Var}\left(\mathbf{V} \mid H_{t}\right)$ is sufficiently close to 0 .

Remark that market makers cannot infer from a trader's order more than what the trader knows, and that a trader's inventory does not provide information about the expectation of $\mathbf{V}$. Thus, from equation (1) we have that at time $t$ the price schedule satisfies

$$
E\left[\mathbf{V} \mid H_{t}, \mathbf{s}=l\right] \leq P_{1}(Q) \leq E\left[\mathbf{V} \mid H_{t}, \mathbf{s}=h\right], \quad \forall Q \in \mathbb{Z}
$$

Let $u_{i}$ be trader $i$ 's utility function that is continuous, strictly increasing and concave. Let denote $v_{t}^{l}=E\left[\mathbf{V} \mid H_{t}, \mathbf{s}=l\right]$, and $v_{t}^{h}=E\left[\mathbf{V} \mid H_{t}, \mathbf{s}=h\right]$. Then equations (5) and (6) still hold after substituting $u_{i}, v_{t}^{h}, v_{t}^{l}$, and 1 to $u, v\left(\pi_{t}^{h}\right), v\left(\pi_{t}^{l}\right)$ and $\delta$ respectively. Moreover, from expression (10) we have

$$
\begin{aligned}
\lim _{\varsigma \rightarrow 0} E\left[u_{i}\left(m+(x+Q) \mathbf{v}-Q v_{t}^{h}\right) \mid H_{t}, s\right] & =\lim _{\varsigma \rightarrow 0} E\left[u_{i}\left(m+(x+Q) \mathbf{v}-Q v_{t}^{l}\right) \mid H_{t}, s\right]= \\
& =E\left[u_{i}\left(m+x E\left[\mathbf{V} \mid H_{t}\right]+(x+Q) \boldsymbol{\varepsilon}\right)\right] .
\end{aligned}
$$

Because of risk aversion, for any constant $V$ it results that

$$
E\left[u_{i}(m+x V+(x+Q) \varepsilon)\right]
$$

is maximized for $Q^{*}=-x$.

If $\operatorname{Var}\left(V \mid H_{t}\right)$ is sufficiently small, then equations (8) and (9) will still hold after substituting $u_{i}, v_{t}^{h}, v_{t}^{l}, 1$ and $-x$ to $u, v\left(\pi_{t}^{h}\right), v\left(\pi_{t}^{l}\right), \delta$ and $\widehat{Q}$ respectively. Thus for $\operatorname{Var}\left(V \mid H_{t}\right)$ sufficiently close to 0 :

$$
\arg \max _{Q \in \mathbb{Z}} E\left[u_{i}\left(m+(x+Q) \mathbf{v}-Q P_{1}(Q)\right) \mid s\right]=-x \quad \forall s \in \Sigma
$$


That means that when public belief are sufficiently precise, traders' order only reflect their inventory concerns and provides no information about $\mathbf{V}$.

Proof of Lemma 2: From Proposition 1 we know that when all traders' orders are not informative, in equilibrium $P_{1}(Q)=v\left(\pi_{t}\right)$ for all $Q$. From Corollary 1 we deduce that when $\pi_{t}$ is sufficiently close to 1 or to 0 and $P_{1}(Q)=v\left(\pi_{t}\right)$, then a trader with inventory $x \in \mathbb{Z}$ will trade exactly $-x$ no matter he received a bullish or a bearish signal. As the expression $E\left[u\left(m+(x+q) \mathbf{v}-v\left(\pi_{t}\right) q\right) \mid \mathbf{H}_{t}, \mathbf{s}\right]$ is a strictly concave function in the traded quantity $q \in \mathbb{R}$, then it will have a unique maximum. Thus in order to find $\bar{\pi}$ (resp. $\underline{\pi}$ ), it is sufficient to find the minimum $\pi_{t}>1 / 2\left(\operatorname{resp} . \operatorname{maximum} \pi_{t}<1 / 2\right)$ such that the trader prefers to trade $-x$ rather than $-x-1$ or $-x+1$ for both $s=h$ and $s=l$. That is to say

$u\left(m+v\left(\pi_{t}\right) x\right)>\max \left\{E\left[u\left(m+\mathbf{v}+(x-1) v\left(\pi_{t}\right)\right) \mid H_{t}, s\right], E\left[u\left(m-\mathbf{v}+(x+1) v\left(\pi_{t}\right)\right) \mid H_{t}, s\right]\right\}$

for $s=h$ and $s=l$. Considering that $u(W)=-e^{-\gamma w}$ and that $\varepsilon \hookrightarrow N\left(0, \sigma_{\varepsilon}\right)$, we have that expression (12) is satisfied only if both inequalities in Lemma 3 are met.

\section{References}

Avery C., P. Zemsky, 1998, "Multidimensional Uncertainty and Herd Behavior in Financial Markets", The American Economic Review, 88, pp. 724-748.

Amihud Y., H. Mendelson and J. Uno, 1999, "Number of Shareholders and Stock Prices: Evidence from Japan", Journal of Finance, 54, pp. 1169-1184.

Biais, B., D. Martimort and J.C. Rochet, 2000, "Competing Mechanisms in a Common Value Environment", Econometrica, 68, 4, 799-837. 
Biais B., L. Glosten, and C. Spatt, 2002. "The Microstructure of Stock Markets". CEPR Discussion Paper no. 3288. London, Centre for Economic Policy Research. http://www.cepr.org/pubs/dps/DP3288.asp.

Bikhchandani S., D. Hirshleifer, I. Welch, 1992, "A Theory of Fads, Fashion, Custom and Cultural Change as Informational Cascades", Journal of Political Economy, 100, pp. $992-1026$.

Chamley C., 2001, "Rational Herds", http://econ.bu.edu/chamley/chamley.html, University of Boston.

Cipriani M. , A. Guarino, 2003, "Herd Behavior and Contagion in Financial Markets", mimeo, New York University.

Décamps J.P., S. Lovo, 2002, "Risk Aversion and Herd Behavior in Financial Markets", HEC CR 758/2002.

Diamond D.W., R.E. Verrechia, 1987, "Constraints on Short-selling and Asset Price Adjustments to Private Information ", Journal of Financial Economics 18, pp. 277-311

Easley D., M. O’Hara, 1992, "Time and the Process of Security Price Adjustment", Journal of Finance, 57, pp. 577-605.

Glosten L., 1989, "Insider Trading, Liquidity and the Role of the Monopolist Specialist", Journal of Business, 62, pp. 211-235.

Glosten L., P. Milgrom, 1985, "Bid, Ask and Transaction Prices in a Specialist Market with Heterogeneously Informed Traders", Journal of Financial Economics, 14, pp. 71100 . 
Gottlieb G and A. Kalay, 1985, "Implication of the Discreteness of Observed Stock Prices", Journal of Finance, 40, pp. 135-153.

Koski J., 1998, "Measurement Effects and the Variance of Returns after Stock Splits and Stock Dividends", Review of Financial Studies, 11, pp. 143-162.

Lamoureux C.G., P. Poon, 1987, "The Market Reaction to Stock Splits", Journal of Finance, 42, pp. 1347-1370.

Lee I. H., 1998, "Market Crashes and Informational Avalanches", Review of Economic Studies, 65, 741-759.

O’Hara M. , 1995, Market Microstructure Theory, Blackwell Publishers.

Ohlson J.A., S. Penman, 1985, "Volatility Increases Subsequent to Stock Splits: An Empirical Aberration", Journal of Financial Economics, 14, pp. 251-266.

Vives X., 1995, "The speed of Information Revelation in a Financial Market Mechanism", Journal of Economic Theory, 67, pp. 178-204. 